\title{
Teams and endoscopy: another effect of the COVID-19 pandemic
}

\author{
Referring to Ravindran S et al. p. 879-883
}

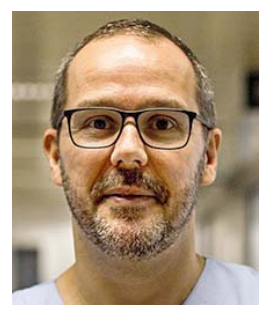

Mário DinisRibeiro

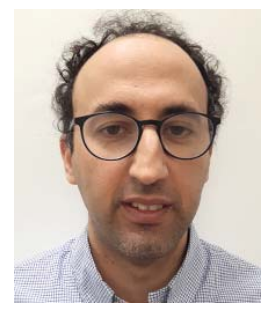

Cesare Hassan

Authors

Mário Dinis-Ribeiro ${ }^{1,2}$, Cesare Hassan ${ }^{3}$

Institutions

1 Gastroenterology Department, Portuguese Oncology Institute of Porto, Porto, Portugal

2 MEDCIDS/CINTESIS, Porto Faculty of Medicine, Porto, Portugal

3 Nuovo Regina Margherita Hospital, Rome, Italy

Bibliography

Endoscopy 2020; 52: 884-885

DOI 10.1055/a-1223-2406

ISSN 0013-726X

(c) 2020. Thieme. All rights reserved.

Georg Thieme Verlag KG, Rüdigerstraße 14,

70469 Stuttgart, Germany

Corresponding author

Mário Dinis-Ribeiro, MD, Instituto Português de Oncologia, Gastrenterologia, Rua Dr. Bernardino de Almedia, Porto 4200-

072, Portugal

Fax: +351-22-5504088

mario@med.up.pt

The quality of healthcare, and in particular gastrointestinal endoscopy, may be perceived differently both by healthcare professionals (HCPs) and by patients according to diverse perspectives or concepts [1]. Quality will vary according to different metrics, but there is usually room for improvement depending on the interventions available at different levels of the endoscopy process.

In 2018, the European Society of Gastrointestinal Endoscopy (ESGE) paid special attention to the quality of the environment in which a patient undergoes an endoscopy procedure. As a clinical community, we somehow need to recognize that, beyond endoscopy performance measures, which clearly depend on the endoscopist, the real or perceived quality of an endoscopy is dependent on other domains that start before and end after the procedure itself. In this regard, the service, and in particular the endoscopy team and the way it is managed, is of paramount importance. In fact, the importance of the leadership team and the defined roles within the team is supported by at least one trial showing a positive impact on endoscopy performance after educational interventions on leadership and endoscopy skills [1].

Human factors play an integral role in teamworking. Teams have a distinct structure but with a "life" that includes goal setting with clear tasks and roles, establishment of working pat- terns, normalization of working styles, and autonomous performance [2]. Not surprisingly, in order to perform adequately, those who lead must embrace some of the key concepts. In endoscopy teams, the key elements relating to patient safety have been identified as human factors, leadership, and communication [3]. Moreover, as training occurs frequently in our endoscopy units, specific team members may change according to rotations. Team processes are interactive, allowing for mutual influence through a series of leading and following interactions [4]. This is opposed to the more traditional concept of leadership found in transformational and transactional leadership theories [5], where teams have an assigned leader whose main responsibility is the achievement of tasks by motivating and rewarding members to overlook self-interest for the greater good of the team's success.

Despite such recommendations, the relevance of the concept of the "endoscopy team" as the foundation for the efficacy and safety of our activity has been somewhat undermined by the prominent role played by individual endoscopist's skills in key quality indicators, such as adenoma detection rate, withdrawal time, and adequacy of tissue sampling during colonoscopy.

Such (apparent) reluctance to adopt a team-based approach has now been recognized as one of the main reasons for the impact on endoscopy units of the global COVID-19 pandemic, 
which started in 2020 [6]. It has been estimated that $5 \%-20 \%$ of HCPs in Western endoscopy units have been infected during the COVID-19 outbreak, in line with the estimated 10\% infection rate among HCPs generally. Such rates are at least 10 -fold higher than similar rates reported in Asian countries, which may be related to different perspectives regarding the team or community versus individuals.

\section{"What is interesting about this toolkit is that it provides a solid and consistent method for long- term implementation of teamworking in endoscopy. What is "new" is the consideration of the human factor as an organizational resource that must be maximized through structured interaction between different healthcare professionals."}

The absolute need to execute the strictest strategies of infection prevention and control (IPC) against COVID-19 has generated the awareness of the importance of the team over the individual. Even a minimal breach of IPC by a single team member may lead to a cluster of COVID-19 infections. Adherence to policies of screening, triage, selection, separation, and monitoring should not be the responsibility of an individual HCP but requires the cooperation of the entire endoscopy team, including nurses, technicians, administrative personnel, and patients: patients must be carefully evaluated for triage and/or testing; COVID-minimization protocols, such as differentiation of endoscopy slots/ rooms according to the risk of infection, must be executed; and availability and proper use of personal protective equipment must be planned. All of this is made more complex by the lack of adequate infrastructure in a substantial proportion of our units. In addition, the profound suppression of any elective endoscopy activity during the 3-month lock-down period has presented the challenge of how to prioritize such burden of examination against an already stressed limited capacity [6, 7].

In this issue of Endoscopy, Ravindran et al. provide a toolkit to support endoscopy teams during the current pandemic (which is not over yet!) [8]. The toolkit aims to help team processes and provides further evidence on its benefit. The adoption of these (or other) ways to consider nontechnical competences in healthcare and endoscopy teams may well be a benefit that we gain from this crisis. All six dimensions explored in the toolkitplanning and anticipating problems, optimizing communication, team cohesion, flattening hierarchy, sharing task burden, and providing support-have clear implications and can be used beyond the current pandemic.

In fact, if we return to the current recommendations for endoscopy during the COVID-19 pandemic [6], which provide direction on indications, how to perform procedures, and how to manage procedures, this guidance will benefit from (more than) daily exercise of leadership. For instance, clear communication, written as well as oral, must occur as in some cases teams are "mirrored;" all professionals must be aligned, and rescheduling of patients, sometimes at diverse timeslots compar- ed with before the pandemic, requires planning and team cohesion; flattening the hierarchy usually poses more burden to leadership but it may bring benefits and sustainable measures.

What is interesting about this toolkit is that it provides a solid and consistent method for long-term implementation of teamworking in endoscopy. What is "new" is the consideration of the human factor as an organizational resource that must be maximized through structured interaction between different HCPs. Such a transparent, proactive, and motivating approach to human interaction has been proven to be extremely effective in reducing the risk of error and improving efficiency within the medical field, such as preventing the administration of incorrect drugs or surgical mismanagements, as well as in other sectors such as the airline industry.

In conclusion, COVID-19 continues to place incredible stress on our endoscopy units. Unexpectedly, it has not impacted the endoscopy skills required to maintain quality assurance, as determined in recent years; however, it has exploited assumptions of our competence to act as a team and our ability to protect ourselves and our patients through shared strategies of IPC. But we have learned the lesson. As we embark on the post-lockdown period, if we can gradually increase our endoscopy capacity, it will be because we implemented a rigorous and structured team approach, with shared leadership and balanced approaches. Hopefully, as the impact of COVID-19 wanes, the strengthened teamworking culture will be a positive long-term effect of the pandemic.

\section{Competing interests}

The authors declare that they have no conflicts of interest.

\section{References}

[1] Valori R, Cortas G, de Lange T et al. Performance measures for endoscopy services: a European Society of Gastrointestinal Endoscopy (ESGE) Quality Improvement Initiative. Endoscopy 2018; 50: 1186-1204

[2] Shah BJ. How to deliver safer and effective patient care: tips for team leaders and educators. Gastroenterology 2019; 156: 852-855

[3] Valori RM, Johnston DJ. Leadership and team building in gastrointestinal endoscopy. Best Pract Res Clin Gastroenterol 2016; 30: 497-509

[4] D'Innocenzo L, Mathieu JE, Kukenberger MR. A meta-analysis of different forms of shared leadership-team performance relations. J Manage 2014; 20: 1-28

[5] Yukl G. An evaluation of conceptual weaknesses in transformational and charismatic leadership theories. Leadersh Q 1999; 10: 285-305

[6] Gralnek IM, Hassan C, Beilenhoff $U$ et al. ESGE and ESGENA Position Statement on gastrointestinal endoscopy and the COVID-19 pandemic. Endoscopy 2020; 52: 483-490

[7] Rodrigues-de-Santiago E, Frazzoni E, Fuccion L et al. Digestive findings that do not require endoscopic surveillance - reducing the burden of care: European Society of Gastrointestinal Endoscopy (ESGE) Position Statement. Endoscopy 2020; 52: 1-7

[8] Ravindran S, Matharoo M, Coleman M et al. Teamworking in endoscopy: a human factors toolkit for the COVID-19 era. Endoscopy 2020; 52: $879-883$ 